\title{
CCD PHOTOMETRY IN THE GLOBULAR CLUSTER M4
}

\author{
W.K. GRIFFITHS, I.N. KANATAS \\ Department of Physics, Leeds University, Leeds, UK \\ AND \\ R.J. DICKENS, A.J. PENNY \\ Rutherford Appleton Laboratory, UK
}

A $V, B-V$ composite colour-magnitude diagram, based upon CCD photometry from $V \sim 12$ on the red giant branch to $V \sim 25$ on the main-sequence has been derived for the globular cluster M4. A distance to the cluster of $(m-M)_{V}=12.84 \pm 0.19$ is determined and the best match to theoretical isochrones is for the case $[\mathrm{Fe} / \mathrm{H}]=-1.27$ and an age of $16 \pm 1 \mathrm{Gyr}$. A differential age comparison with NGC 362 shows that M4 is approximately 1.7 Gyr older.

The luminosity function of the cluster has been constructed and appears to reach $M_{V}>11$. At $M_{V}>10$ the luminosity function shows a turnover. The resulting mass function extends to $\mathcal{M} \sim 0.19 \mathcal{M}_{\odot}$ and is quite flat having a power-law index of $x=-0.15 \pm 0.04$. It is suggested that the most likely explanation for the observed flatness in the mass function is that the cluster has undergone extensive tidal stripping.

The spread in metallicity amongst the stars in the cluster is limited to \pm 0.09 dex by the small scatter on the subgiant branch.

The telescopes used were the Anglo-Australian Telescope (AAT) and the South African Astronomical Observatory (SAAO) 1-m telescope. The data were reduced predominantly with the stellar photometry (image and table-handling) package STARMAN, developed by A.J. Penny (1992). This has a full reduction capability, and has powerful programs for dealing with stars in crowded fields.

\section{References}

Kanatas, I.N., Griffiths, W.K., Dickens, R.J., Penny, A.J., 1994, MNRAS, in press.

Penny, A.J., 1992, Starman User Manual Version 1.0. Starlink User Note 141.1, Starlink Project, Rutherford Appleton Laboratory. 